\title{
Imagerie multimodale en choriorétinopathie séreuse centrale aiguë et chronique
}

\author{
An Vo, D.O. \\ Professeur adjoint \\ Western University \\ of Health Sciences College \\ of Optometry \\ Jessica Haynes, D.O., F.A.A.O. \\ Optométriste associée \\ Charles Retina Institute; \\ Southern College \\ of Optometry \\ Faculté auxiliaire
}

\begin{abstract}
Résumé
De nombreuses théories ont été proposées concernant la pathophysiologie de la choriorétinopathie séreuse centrale (CRSC), sans consensus sur son étiologie exacte. La recherche a mené à diverses possibilités de traitement qui ciblent des mécanismes précis. L’imagerie multimodale, y compris l'autofluorescence du fond de l'œil (FAF), la tomographie par cohérence optique (TCO), l'angiographie fluorescéinique et l'angiographie au vert d'indocyanine, identifie les altérations vasculaires et structurelles qui peuvent confirmer le diagnostic de CRSC et adapter efficacement le traitement pour aider à maximiser le pronostic visuel chez ces patients. Les caractéristiques d'imagerie de la CRSC et des exemples de cas seront explorés.
\end{abstract}

\section{MOTS CLÉS}

Rétinopathie séreuse centrale, choriorétinopathie séreuse centrale, CRSC, RSCC

\section{INTRODUCTION}

La rétinopathie séreuse centrale est une condition relativement courante caractérisée par des zones localisées de détachement neurosensoriel de la rétine, le plus souvent dans la macula, avec ou sans détachement de l'épithélium pigmentaire rétinien (EPR). ${ }^{1}$ La présentation des patients varie, avec des symptômes courants comme une légère rougeur, une métamorphopsie, une dyschromatopsie et une sensibilité au contraste réduite. La CRSC est généralement unilatérale, mais elle peut se manifester bilatéralement, bien qu'elle soit presque exclusivement asymétrique. ${ }^{2}$

La CRSC est la quatrième maladie visuelle la plus courante dans le monde après la dégénérescence maculaire, la rétinopathie diabétique et l'occlusion veineuse rétinienne. ${ }^{3,4} \mathrm{Chez}$ les hommes, l'incidence moyenne mondiale est de 10 pour 100 000, et les hommes sont six fois plus susceptibles d'être touchés que les femmes. Il y a une légère prédilection raciale chez les Asiatiques et les Blancs par rapport à ceux d'origine africaine, bien que la CRSC chez les Afro-Américains ait été associée à un résultat visuel plus médiocre et à une présentation plus agressive. ${ }^{2,4}$ 'âge moyen au moment du diagnostic varie entre 39 et 51 ans, et les patients de plus de 50 ans présentent un risque plus élevé de néovascularisation choroïdienne et un traitement prolongé. ${ }^{4}$

Dans les cas aigus, la CRSC se résout souvent sans intervention. Plusieurs options de traitement ont été proposées pour les cas chroniques, y compris la photocoagulation laser focale, la thérapie photodynamique (TPD), l'injection d'un facteur de croissance endothélial antivasculaire (antiVEGF), la thérapie anticorticostéroïde, les inhibiteurs de l'aldostérone comme l'éphérone et l'arrêt de l'utilisation de stéroïdes systémiques. ${ }^{5}$ 
L'efficacité d'options de traitement comme l'antiVEGF, la thérapie anticorticostéroïde et les inhibiteurs de l'aldostérone demeure controversée. De plus, ce ne sont pas toutes les présentations de CRSC qui se prêtent à la thérapie au laser. Ainsi, la prise en charge d'un patient avec CRSC devient très individualisée. Il est crucial de poser un diagnostic correct de la maladie et de procéder à une évaluation par imagerie multimodale pour fournir des soins appropriés aux patients. Les cas suivants explorent les caractéristiques diagnostiques des patients atteints de CRSC à l'aide de l'imagerie TCO, de l'autofluorescence du fond de l'œil, de l'angiographie fluorescéinique et de l'angiographie au vert d'indocyanine.

TOMOGRAPHIE PAR COHÉRENCE OPTIQUE (TCO)

L'avènement de l'imagerie TCO a permis aux cliniciens de détecter précisément la CRSC et de suivre son évolution clinique en identifiant les caractéristiques de la choroïde et de la rétine. La constatation clinique la plus identifiable en matière de CRSC est le détachement rétinien séreux. Sur l'imagerie TCO, cela apparaît comme une séparation optique vide de la rétine neurosensorielle de l'EPR impliquant la macula (figure 1). De plus, un détachement épithélial pigmenté (DEP) peut se présenter comme une séparation focale de l'EPR par rapport à la membrane sous-jacente de Bruch dans le décollement rétinien séreux. Cette constatation peut être importante ou subtile et est considérée comme le principal point d'entrée du liquide qui fuit dans l'espace sous-rétinien (figure 1). ${ }^{6}$

Figure 1 : Deux patients dont la CRSC montre des décollements rétiniens séreux (flèches jaunes) avec des DEP (flèches rouges). Les DEP peuvent être gros et proéminents comme chez le patient A, ou subtils et aplatis comme chez le patient $B$.
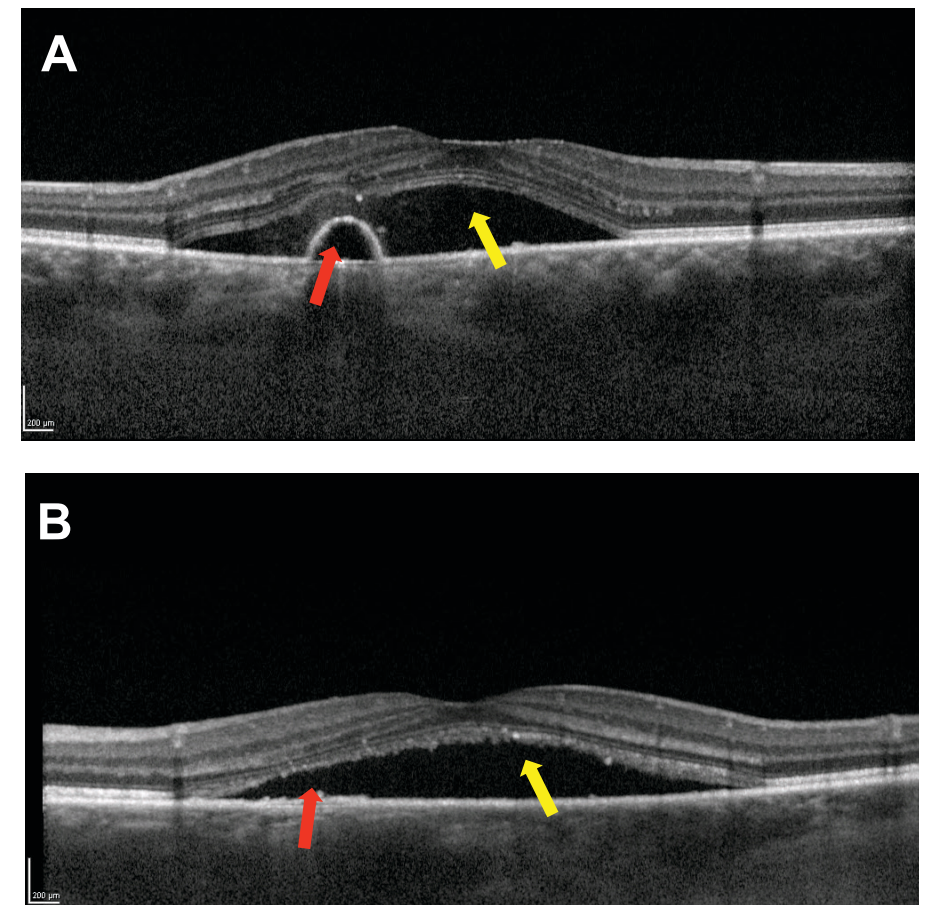

L’imagerie en profondeur améliorée (EDI) permet d'améliorer l'imagerie de la choroïde et la mesure reproductible de l'épaisseur choroïdienne. Cette stratégie d'imagerie par TCO est très utile pour l'évaluation des patients atteints de CRSC, puisque l'épaisseur choroïdienne accrue est un résultat bien établi chez la plupart des patients atteints de la maladie (figure 2). L'EDI a également mené à l'avènement de l'indice vasculaire choroïdien (IVC), qui est le rapport entre la surface lumineuse, ou zone vasculaire de la choroïde, et la superficie totale de la choroïde. On a signalé que les cas aigus de CRSC présentaient un IVC moyen plus élevé que les patients d'âge correspondant ou atteints de CRSC chronique. ${ }^{6}$ Les patients atteints de CRSC associé à des stéroïdes avaient aussi tendance à présenter un IVC moyen plus élevé que les cas idiopathiques. ${ }^{7}$ Bien que l'IVC soit actuellement utilisé principalement dans les milieux de recherche, il se peut qu'à l'avenir, il s'avère utile pour comprendre la pathophysiologie de la CRSC dans la pratique générale.

La mesure de l'épaisseur choroïdienne est indiquée pour un large éventail de conditions, surtout en ce qui concerne les conditions du spectre pachychoroïdien. Les conditions considérées comme faisant partie de ce spectre partagent toutes une choroïde plus épaisse que la moyenne et comprennent l'épithéliopathie pigmentaire pachychoroïdienne, 
la CRSC et la vasculopathie choroïdienne polypoïde. ${ }^{8}$ Les mesures sont généralement prises directement sous la fovéa pour assurer la répétabilité. Les valeurs normatives pour l'épaisseur choroïdale subfovéale dans la littérature varient de 272 à $448 \mu \mathrm{m}$, car l'épaisseur varie en fonction de l'erreur de réfraction, de l'âge et de l'origine ethnique, bien que les valeurs supérieures à $400 \mu \mathrm{m}$ devraient éveiller des soupçons. ${ }^{9}$ Par exemple, une étude menée en 2018 auprès de 104 adultes iraniens normaux a révélé une épaisseur moyenne choroïdale subfovéale de $363 \pm 84 \mu \mathrm{m}$, tandis qu’une autre étude menée en 2008 auprès d'adultes américains a révélé une épaisseur moyenne subfovéale de $287 \pm 76 \mu$ m. ${ }^{10,11}$ Les patients qui ont une CRSC active présentent une épaisseur choroïdienne subfovéale plus grande que leurs valeurs résolues. Chung et coll. en Corée du Sud ont constaté une épaisseur moyenne de 446,8 \pm 101,0 dans les cas actifs par rapport à 414,7 $\pm 93,9$ dans les cas résolus. ${ }^{12}$ Les tendances générales à prendre en considération comprennent l'amincissement avec l'âge et l'amincissement avec une longueur axiale accrue.

Figure 2: TCO avec imagerie en profondeur améliorée révélant une épaisseur choroïdienne supérieure à la moyenne chez un patient présentant une CRSC.

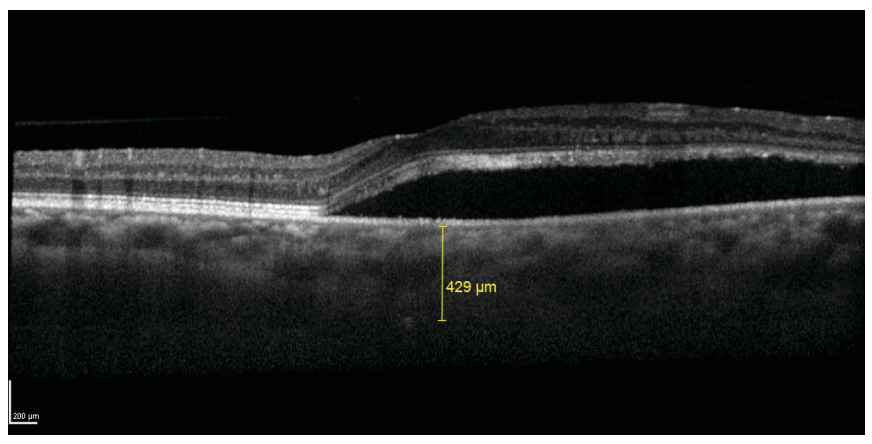

L'imagerie TCO peut également fournir des indices diagnostiques de la chronicité de la maladie. Dans le cas d'un détachement séreux chronique, les photorécepteurs restent détachés de l'EPR pendant une période prolongée, ce qui entraîne une phagocytose inadéquate de leurs segments extérieurs. L'allongement des segments extérieurs mène à la constatation TCO appelée « photorécepteurs fragmentés » (figure 3 ). ${ }^{13} \mathrm{Il}$ est important de noter que les photorécepteurs fragmentés ne sont pas uniques à la CRSC et sont également visibles dans d'autres formes de détachement chronique, comme les détachements séreux chroniques du mélanome choroïdien. ${ }^{14}$

En plus des photorécepteurs fragmentés, les patients atteints d'une maladie chronique peuvent développer une atrophie géographique et un œdème rétinien kystique qui se distingue par l'imagerie TCO. Ce sont là des indices d'un problème de longue date. Dans le cas d'un important EPR central et d'une atrophie des photorécepteurs, un traitement plus agressif peut être inutile, puisque le pronostic visuel demeure faible, peu importe l'intervention.

\section{TCO-ANGIOGRAPHIE}

Les patients qui ont une rétinopathie séreuse centrale risquent également de développer des membranes néovasculaires choroïdiennes (MNV), les estimations variant entre 2 et $9 \%$ des cas chroniques. ${ }^{15}$ Il peut être difficile de déterminer l'origine du liquide apparent, que ce soit à partir de la maladie elle-même ou du développement du MNV. La capacité d'effectuer une imagerie détaillée avec TCO est utile dans ces cas. L'imagerie TCO-angiographie (TCO-A) est une méthode non invasive de détection des altérations pathologiques dans le système vasculaire sans injection de colorant. La TCO-A fournit des renseignements structuraux vitaux profonds à l'EPR qui sont typiques de l'imagerie TCO conventionnelle, ainsi qu'une analyse ciblée des caractéristiques anormales du débit sanguin, avec une sensibilité semblable à celle de l'angiographie fluorescéinique. ${ }^{15}$ Le praticien peut cibler la zone de MNV présumée par TCO et évaluer la choriocapillaire sous-jacente pour la néovascularisation. Le MNV est de plus en plus bien caractérisé en TCO-A et son utilité pourrait augmenter en raison de sa sécurité et de sa facilité d'utilisation par rapport à l'angiographie fluorescéinique. La TCO-A peut également s'avérer utile dans les cas où il n'y a pas de néovascularisation. Une étude récente a fourni des preuves de l'hyperperfusion et de l'hypoperfusion au niveau de la choriocapillaire dans les régions de détachement séreux, ainsi que de la régression de la perfusion après la résolution du liquide sous-rétinien. ${ }^{9}$ Cela porte à croire que la TCO-A peut être utilisée pour évaluer le risque de progression des CRSC en surveillant le profil des anomalies du débit. 
AUTOFLUORESCENCE DU FOND DE L'GIL

L'imagerie autofluorescente du fond de l'œil est un outil utile dans l'évaluation de la CRSC. Cette technique permet de recueillir des données sur les émissions de lumière en utilisant l'excitation à la lumière bleue pour visualiser la teneur en fluorophore (mélanine ou lipofuscine) dans toute la rétine. Le FAF est utilisé pour identifier les drusen et les anomalies de la rétine externe et de l'EPR qui seraient autrement difficiles à détecter lors de l'examen du fond de l'œil, de la photographie du fond de l'œil ou de l'angiographie fluorescéinique. ${ }^{16,17}$ Les anomalies sont différenciées les unes des autres par leur intensité de signal, notamment l'hyper-autofluorescent, l'hypo-autofluorescent et l'iso-autofluorescent.

Figure 3 : A. Détachement séreux aigu causé par la CRSC avec allongement minimal des segments extérieurs du photorécepteur (flèche jaune); B et C. Décollements séreux chroniques avec découverte de "photorécepteurs fragmentés » (flèches rouges) causés par l'allongement des segments extérieurs.

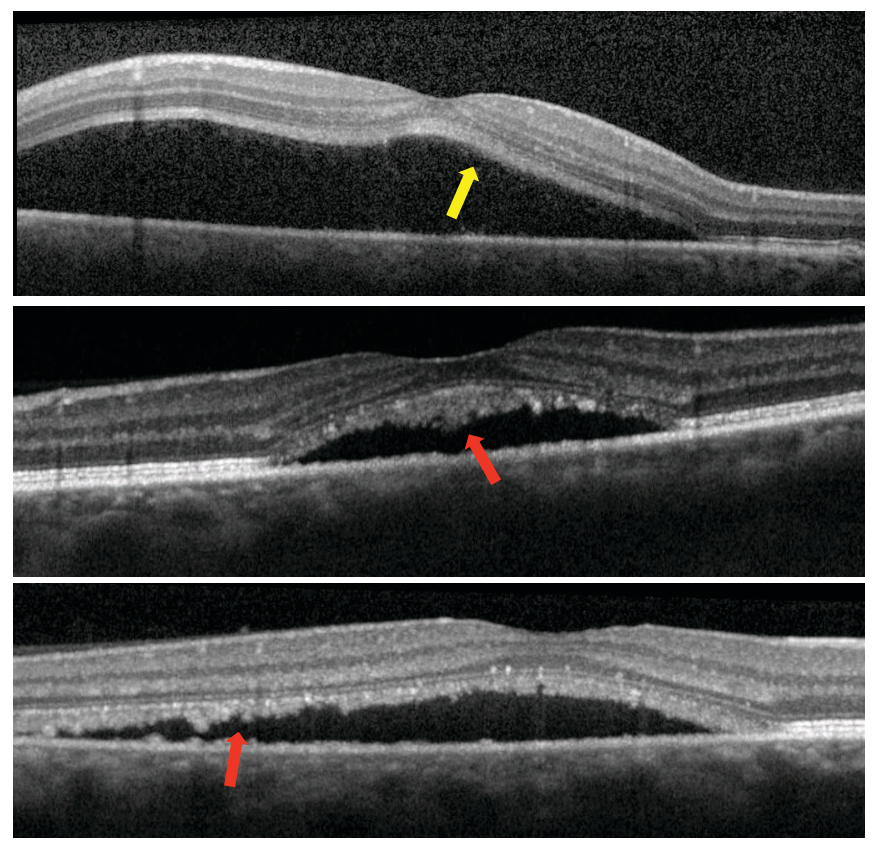

Dans les cas aigus de CRSC, le liquide sous-rétinien accumulé peut créer un effet de blocage qui entraîne une région d'hypofluorescence (figure 4). L'étendue du détachement séreux peut être plus facilement visualisée dans le FAF que dans l'évaluation clinique. Dans les cas chroniques, localiser les zones d'hyper-autofluorescence. On pense qu'il s'agit de précipités de fluorophores provenant de segments extérieurs de photorécepteurs non phagocytés et allongés (figure 4). Au fur et à mesure que la condition devient très ancienne ou récurrente, des dommages à l'EPR peuvent se produire, car les deux régions d'hyper-autofluorescence sont corrélées à des tissus non viables et à des fluorophores accumulés et à des régions d'hypofluorescence correspondant aux régions où l'atrophie de l'EPR s'est déjà produite. ${ }^{17} \mathrm{Dans}$ le cas des maladies chroniques, la perturbation de l'EPR sur le FAF peut prendre une apparence inhabituelle, car le liquide séreux est déplacé vers le bas par la gravité, ce qui entraîne de l'hyper-autofluorescence en « queue de comète ».13

\section{ANGIOGRAPHIE FLUORESCÉINIQUE}

L'angiographie fluorescéinique est utile pour identifier les zones de fuite, surtout dans les cas où plusieurs détachements se trouvent à l'extérieur de la macula. La fuite peut se produire dans un motif de tache d'encre qui commence par un point focal et s'agrandit dans la phase tardive (figure 5). Les fuites peuvent également se manifester sous la forme d'une cheminée de fumée semblable à l'œdème maculaire cystoïde, qui commence également comme point focal, mais croît sous une forme semblable à celle des nuages jusqu'à la phase tardive (figure 6). La forme d'une cheminée de fumée n'est pas aussi courante dans les CRSC et apparaît dans 10 à $15 \%$ des cas aigus. ${ }^{2}$ L'identification des DEP par angiographie fluorescéinique peut être vitale, surtout lorsqu'on envisage la coagulation laser focale. Si la zone de fuite est à une distance suffisante de la fovéa pour être considérée comme sécuritaire, l'application au laser peut raccourcir la durée de deux mois et peut réduire le taux de récurrence. ${ }^{18,19}$ La photocoagulation au laser, telle que le système Navilas (OD-OS GmbH, Teltow en Allemagne), permet de suivre l'œil et de planifier le traitement pré-rendu en travaillant en conjonction avec la photographie du fond de l'œil et l'angiographie fluorescéinique, et s'est révélée plus précise que le laser conventionnel. ${ }^{19} \mathrm{Il}$ se peut toutefois que l'utilisation précoce du laser ne soit pas indiquée dans tous les cas et qu'elle soit précédée d'une période d'observation au-delà de la période de 3 à 4 mois. ${ }^{20}$ 
Figure 4 : A. Patient présentant une CRSC aiguë et une région bien circonscrite d'hypoautofluorescence assez uniforme qui correspond à la zone de détachement séreux; B. Patient présentant une CRSC chronique et des photorécepteurs fragmentés sur la TCO, qui révèle les points de fuite résultant de l'hyperautofluorescence.

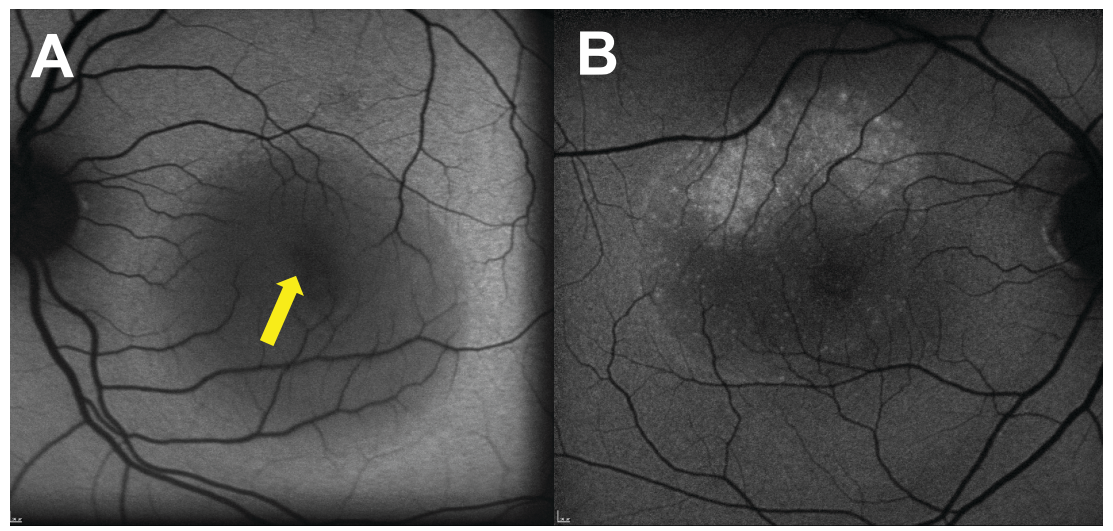

Figure 5 : A. Point de fuite précoce à 30 secondes; B. Augmentation de l'hyperfluorescence en tache d'encre au cours des phases ultérieures; C. Zone de fuite correspondant au DEP observé sur la TCO.
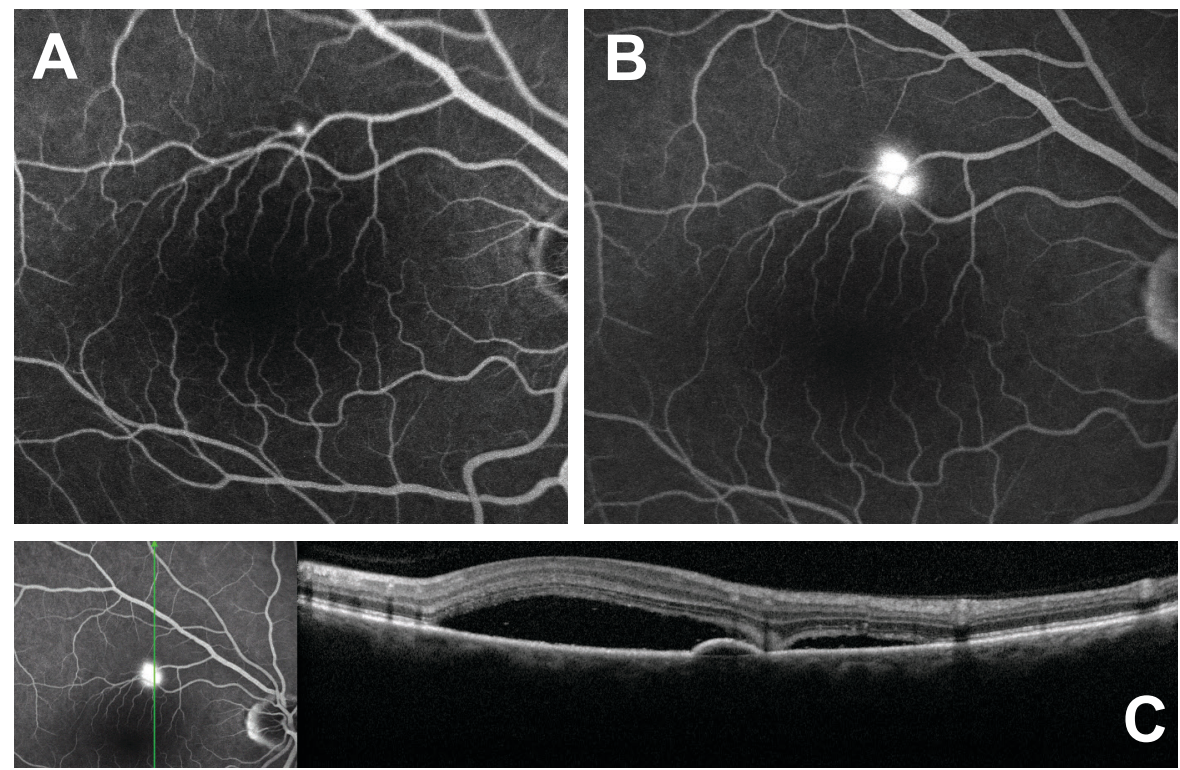

Figure 6 : Apparence de cheminée des fuites.
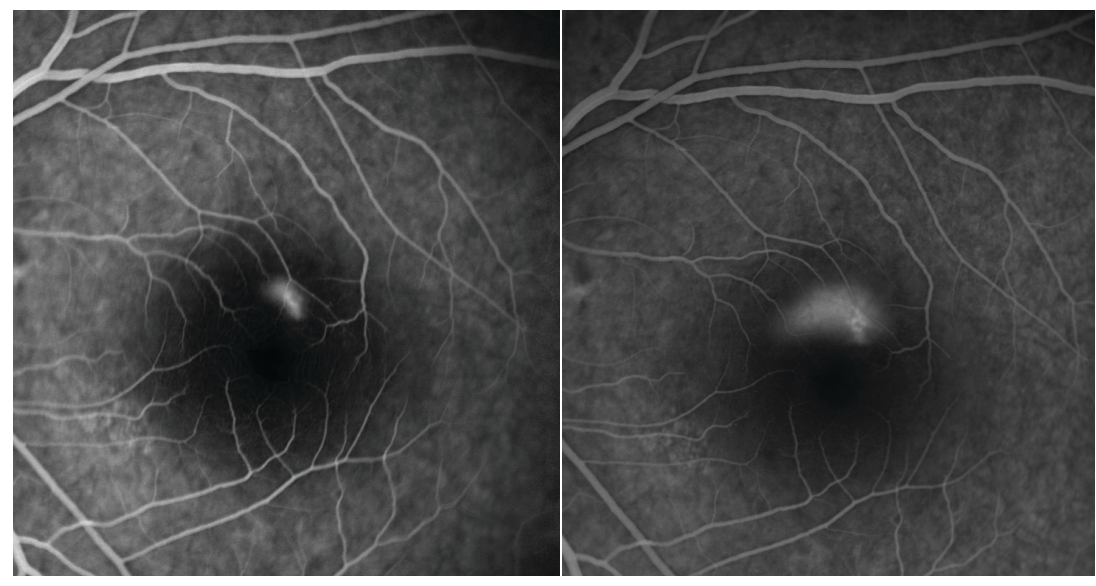


\section{ANGIOGRAPHIE AU VERT D'INDOCYANINE}

L'angiographie au vert d'indocyanine (VIC) est une autre méthode d'imagerie intraveineuse qui peut être réalisée en même temps que l'angiographie fluorescéinique et met en évidence les changements vasculaires choroïdiens. Le VIC présente une liaison plasmatique de près de $98 \%$, ce qui entraîne une rétention beaucoup plus importante du colorant que la fluorescéine dans le vasculaire choroïdien qui fuit autrement. ${ }^{2}$ L'utilisation du VIC est particulièrement utile en combinaison avec la thérapie photodynamique (TPD), puisque la visualisation accrue de la vasculaire choroïdienne permet de cibler plus précisément les fuites. Dans la CRSC, il y a un remplissage choroïdal retardé dans la phase précoce et des plaques de non-perfusion, ce qui entraîne une dilatation veineuse choroïdienne et une hyperfluorescence géographique dans la phase intermédiaire (figures 7 et 8). ${ }^{21}$ Ces zones de choroïde hyperperméable sont en corrélation avec les zones de fuite de l'EPR, mais peuvent également apparaître ailleurs dans la rétine. ${ }^{2}$ Cette anomalie dans le remplissage vasculaire est souvent observée bilatéralement et plus largement sur le pôle postérieur, par rapport aux changements focaux observés dans l'imagerie par fluorescéine. Il faut procéder au VIC pour exclure la vasculopathie choroïdienne polypoïde (VCP) et les autres causes de néovascularisation. La VCP, caractérisée par des vaisseaux choroïdiens anormaux qui culminent en projections focales dans la rétine postérieure, c'est-à-dire la zone qui entoure le nerf optique ou la macula, peut être très similaire à la CRSC sous autofluorescence, mais elle est mieux caractérisée par le VIC comme ayant davantage de lésions ayant un aspect de polype entre les phases intermédiaire et tardive. ${ }^{2}$

Figure 7 : A. Angiographie au vert d'indocyanine à la 30e seconde montrant une hypofluorescence centrale avec des vaisseaux choroïdiens dilatés; $B$. Phase tardive de l'angiographie au vert d'indocyanine à la 14e minute montrant une zone localisée d'hyperfluorescence avec possibilité de cibler la région avec un laser focal.
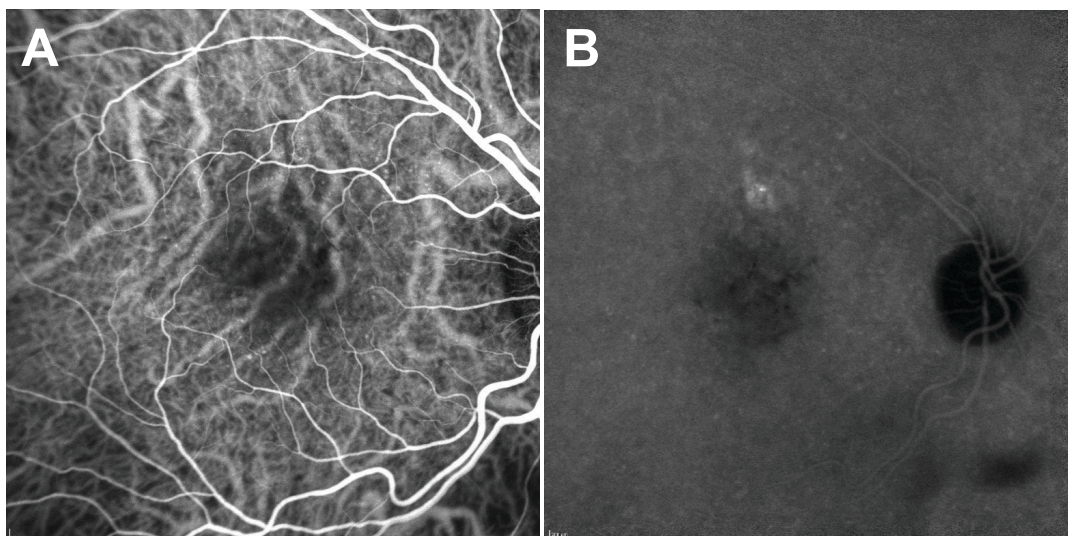

Figure $8:$ A. Phase précoce de l'angiographie au vert d'indocyanine montrant les régions d'hypofluorescence et les vaisseaux chorö̈diens dilatés; $B$. Phase tardive de l'angiographie au vert d'indocyanine montrant les régions de fuite. Les régions plus vastes ou les régions à proximité de la fovéa ne peuvent pas être ciblées par un laser focal, mais peuvent être traitées par TDP.
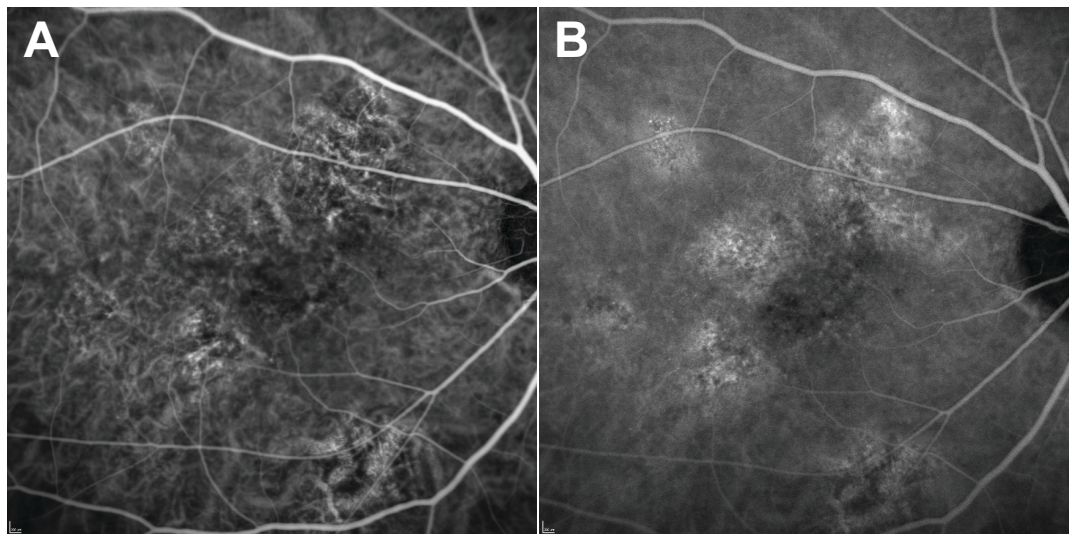

CAS 1

Un Afro-Américain de 65 ans s'est plaint d'une très mauvaise vision à l'œil droit et d'un léger brouillard à l'œil gauche avec ses lunettes habituelles. Il a déclaré que l'œil droit avait une mauvaise vision depuis 15 ans et que plusieurs médecins de l'œil lui avaient déjà dit que sa vision ne s'améliorerait pas avec le traitement. Il a nié tout 
antécédent de traumatisme et s'est souvenu vaguement d'un diagnostic de « dégénérescence maculaire » dans l'œil droit causant sa perte de vision. Il a nié tout antécédent de traitement.

Il avait subi des traitements de chimiothérapie et de radiothérapie pour une lésion non précisée du cerveau et pour un cancer du poumon au cours de l'année précédente et avait l'impression que sa vision avait diminué au cours de cette période. De ses antécédents médicaux ressortent du diabète de type 2 diagnostiqué 3 ans auparavant avec un $\mathrm{HbAlc}$ récent de 6,3\%, de l'hypertension et des antécédents d'accident cérébrovasculaire mineur 5 ans auparavant. Le patient a nié avoir des antécédents de maladie oculaire dans la famille et a nié avoir consommé du tabac, de l'alcool ou des drogues à des fins récréatives. Les médicaments du patient comprenaient notamment $10 \mathrm{mg}$ de cetirizine au besoin, $50 \mathrm{mg}$ de sildénafil au besoin, 2,5 $\mathrm{mg}$ d'apixaban deux fois par jour, 1000 IU de cholécalciférol par jour, de $10 \mathrm{mg}$ hydrocodone/ $325 \mathrm{mg}$ d'acétaminophène (Norco) par jour, $500 \mathrm{mg}$ de levetiracetam deux fois par jour, $50 \mathrm{mg}$ de losartan deux fois par jour, $50 \mathrm{mg} /$ jour de métoprolol, $5 \mathrm{mg} / \mathrm{jour}$ de tradjenta, $20 \mathrm{mg}$ de oméprazole le matin, 0,4 $\mathrm{mg}$ de tamsulosine chaque soir et $1 \mathrm{mg}$ de dexaméthasone deux fois par jour. Il était allergique à la morphine.

Il avait été vu dans notre clinique 16 ans auparavant, et les dossiers indiquaient un diagnostic de dégénérescence maculaire non spécifiée avec des drusen durs et des soupçons d'œdème maculaire. Sa principale plainte au cours de cette visite était une vision floue aiguë des deux yeux (OU) au réveil cinq jours auparavant. La meilleure acuité visuelle corrigée était 20/70- OD et 20/25+ OS. Il devait faire l'objet d'un suivi par angiographie fluorescéinique, mais il n'est pas revenu.

Au cours de sa visite, la vision était de compter les doigts à 3 pieds au niveau OD et de 20/30-2 au niveau OS, sans amélioration de l'acuité visuelle avec trou sténopéique ou réfraction. Les muscles extraoculaires présentaient une gamme complète de mouvements dans les deux yeux sans douleur ni diplopie.

La pression intraoculaire était de $16 \mathrm{mmHg}$ dans les deux yeux. L'examen du segment antérieur a révélé la présence de cataractes sclérotiques nucléaires de grade 2 dans chaque œil, mais n’a rien révélé d'autre. Il n'y avait pas de néovascularisation de l'iris dans aucun des yeux. L'évaluation des segments postérieurs dilatés a révélé des nerfs optiques sains et symétriques sans pâleur avec un ratio C/D de 0,40 rd OD et 0,45 rd OS. Le vitré était clair et la rétine périphérique intacte. La tortuosité vasculaire des artères et des veines OU a été démontrée. Il n’y avait aucun signe de rétinopathie diabétique OU.

L'œil droit a révélé un défaut de « fenêtre » de l'EPR dans la fovéa, d'un diamètre pupillaire d'environ 3/4. La lésion était bien délimitée et encerclée par un mince anneau qui s'étendait vers le bas jusqu'aux arcades inférieures avec des précipités jaunes qui ressemblaient à des exsudats. Dans l'œil gauche, l'arcade supérieure et la macula présentaient des dépôts ressemblant à des drusen (figure 9).

Figure 9 : Photographie du fond de l'œil et imagerie autofluorescente connexe.

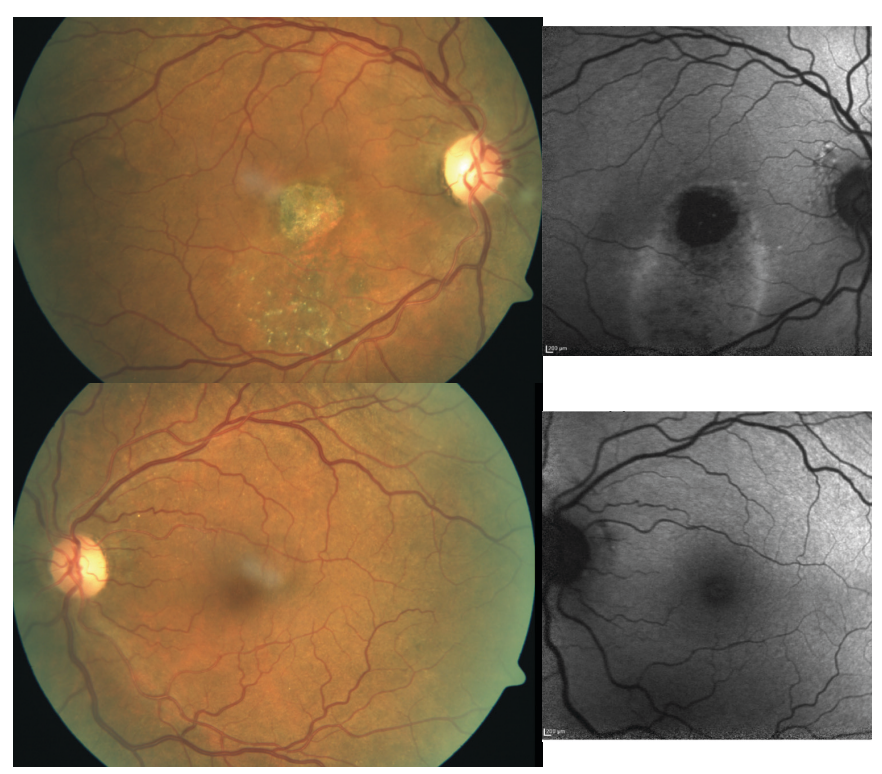


Figure $10: A$. Edème kystique recouvrant la région du photorécepteur et atrophie de l'EPR dans la fovéa; B. DEP peu profond (flèche jaune); C. Atrophie rétinienne externe s'étendant de façon inférieure; D. TCO maculaire normale dans l'œil gauche.
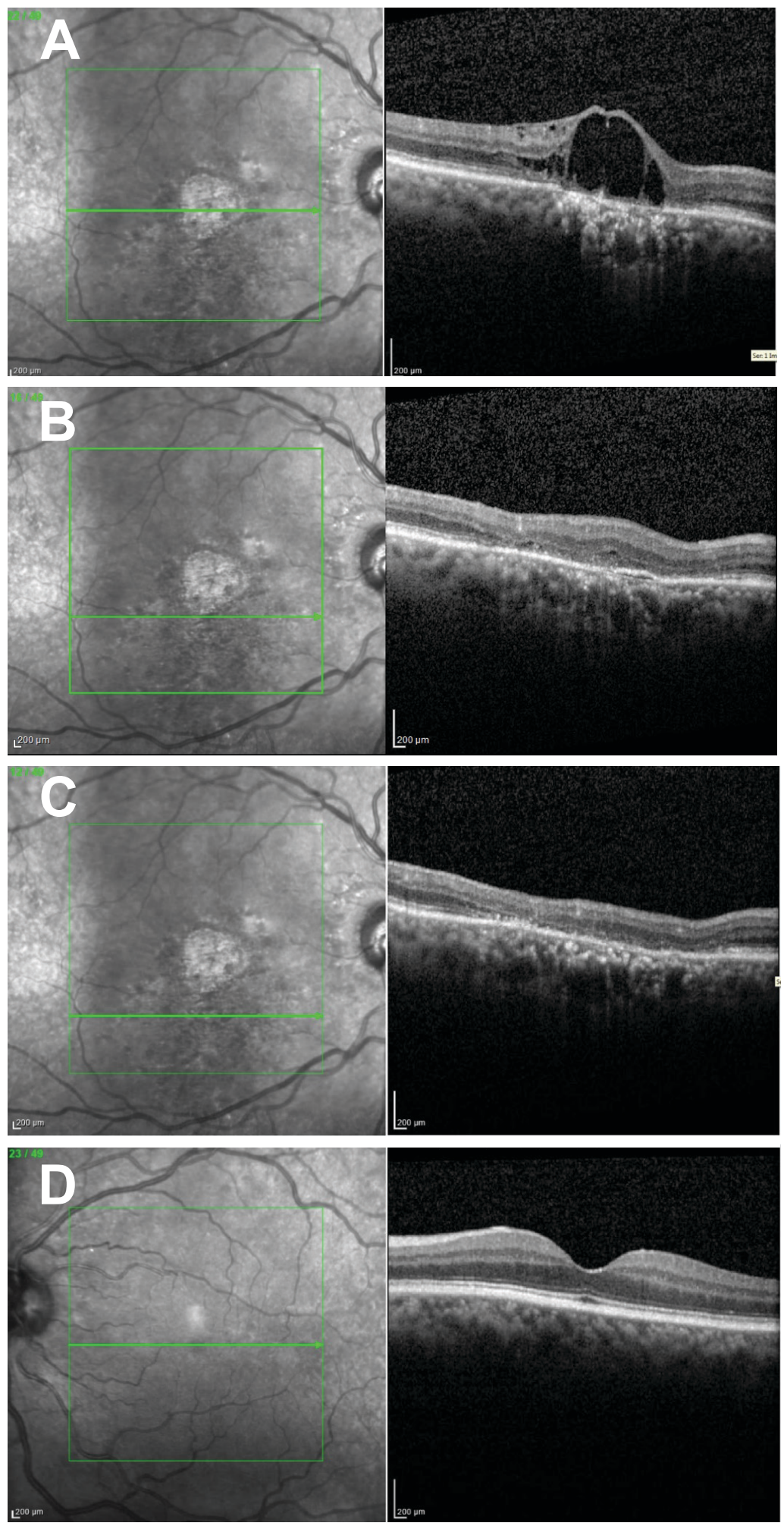
L'autofluorescence du fond de l'œil (FAF) OD a démontré une hypofluorescence dense et homogène de la lésion fovéale correspondant à une atrophie complète de l'EPR. En plus de cette zone, les dommages causés par l'EPR s'étendaient de façon inférieure dans un schéma hyper-autofluorescent clairement défini indiquant un tractus gravitationnel vers l'arcade inférieure. Il y avait également des points hypofluorescents représentatifs des changements pseudo-exudatifs dans l'anneau. Le FAF OS a démontré une pigmentation maculaire même isofluorescente (figure 9).

L'imagerie de la tomographie par cohérence optique SD (TCO) de la macula OD a démontré des altérations kystiques de la fovéa qui ont altéré l'anatomie rétinienne intérieure et externe avec une atrophie externe importante de la rétine et du photorécepteur en dessous. Un petit détachement superficiel de l'EPR (DEP) était manifestement inférieur à la fovéa ainsi qu'une perte rétinienne externe prononcée qui s'étendait aussi loin antérieurement que la couche nucléaire interne et à travers l'EPR. La TCO de la macula OS ne révélait aucun œè̀me et les couches rétiniennes sont demeurées intactes avec la préservation du contour fovéal.

Les résultats du patient concordaient avec la rétinopathie séreuse centrale chronique OD. Le patient a reçu une formation sur la maladie et le pronostic visuel est toujours médiocre malgré le traitement. On lui a conseillé d'éviter les déclencheurs de la CRSC comme l'utilisation de stéroïdes, y compris les vaporisations nasales. Cependant, nous avons recommandé que le patient continue d'utiliser la dexaméthasone, car elle était un complément à sa chimiothérapie. Le patient désirait toutefois une consultation sur les options de traitement et a été recommandé à un surspécialiste de la rétine pour évaluation. Le patient a refusé la dilatation lors de son suivi auprès du rétinologue et n'est pas revenu pour un suivi.

\section{CAS 2}

Un Afro-Américain de 38 ans présentait une tache brune dans sa vision centrale OD, qui avait commencé une semaine auparavant. Il a décrit sa vision comme s'il regardait à travers une tache de café. L'endroit ne bougeait pas et suivait sa fixation. Le patient a nié avoir des symptômes dans l'œil gauche. Il a nié avoir des antécédents de maladie oculaire, de blessure ou de conditions anormales pour lui-même et sa famille immédiate. Son dossier médical a révélé de l'hypertension et du TDAH. Ses médicaments actuels comprenaient $5 \mathrm{mg}$ d'amphétamine $/ 5 \mathrm{mg}$ de dextroamphétamine par jour, $40 \mathrm{mg}$ d'atomoxétine par jour et $500 \mathrm{mg}$ de naproxène deux fois par jour. Les pupilles, les MEO et les champs visuels de confrontation étaient sans particularité OD, OS. La meilleure acuité visuelle corrigée était 20/20- OD et 20/20 OS. Les tests de grilles d'Amsler ont révélé une tache brune centrale et une fixation nasale à une fixation nasale.

La pression intraoculaire était de $18 \mathrm{mmHg}$ OD et $20 \mathrm{mmHg}$ OS. L'examen du segment antérieur n'a rien révélé d'anormal, OD, OS. L'examen dilaté du segment postérieur a démontré un grand anneau non pigmenté et circonscrit autour de la fovéa OD, qui était plus grand dans la partie inférieure (figure 10). La macula OS n'avait rien d'anormal.

Le rapport C/D des nerfs optiques était de 0,30 OD et OS. La périphérie ne révélait aucun trou, aucune rupture ou déchirure et le système vasculaire était de calibre normal dans les deux yeux.

L'imagerie TCO de la macula OD a révélé un détachement neurosensoriel de 8 à 10 diamètres pupillaires allant de la macula temporale à la zone située tout juste sous la fovéa (figures 11,12). La TCO a démontré une épaisseur choroïdale subfovéale supérieure à 450 microns (à l'extérieur de la plage normative acceptée) et un petit DEP présent dans le détachement séreux ${ }^{27}$. Les segments extérieurs du photorécepteur étaient très peu allongés.

Figure 11 : Conclusions d'examen du fond d'œil de l'œil droit.

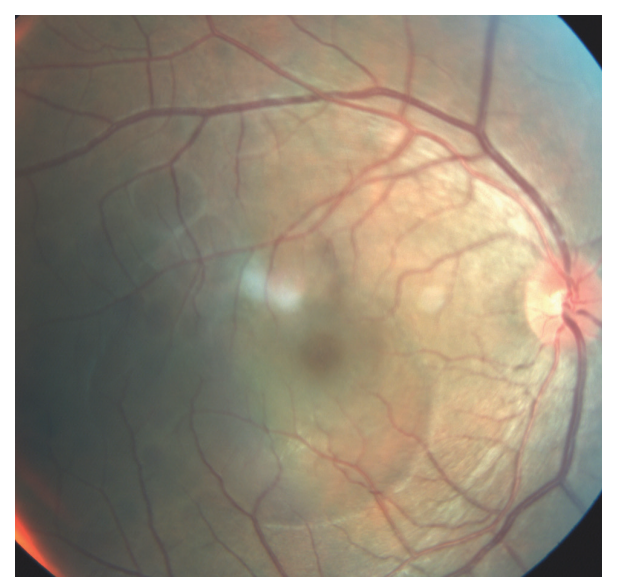


Figure 12 : Comparaison de la progression des tranches horizontales avec la TCO maculaire. A. Liquide subrétinien persistant sur une période de 3 mois d'une région inférieure à la macula.
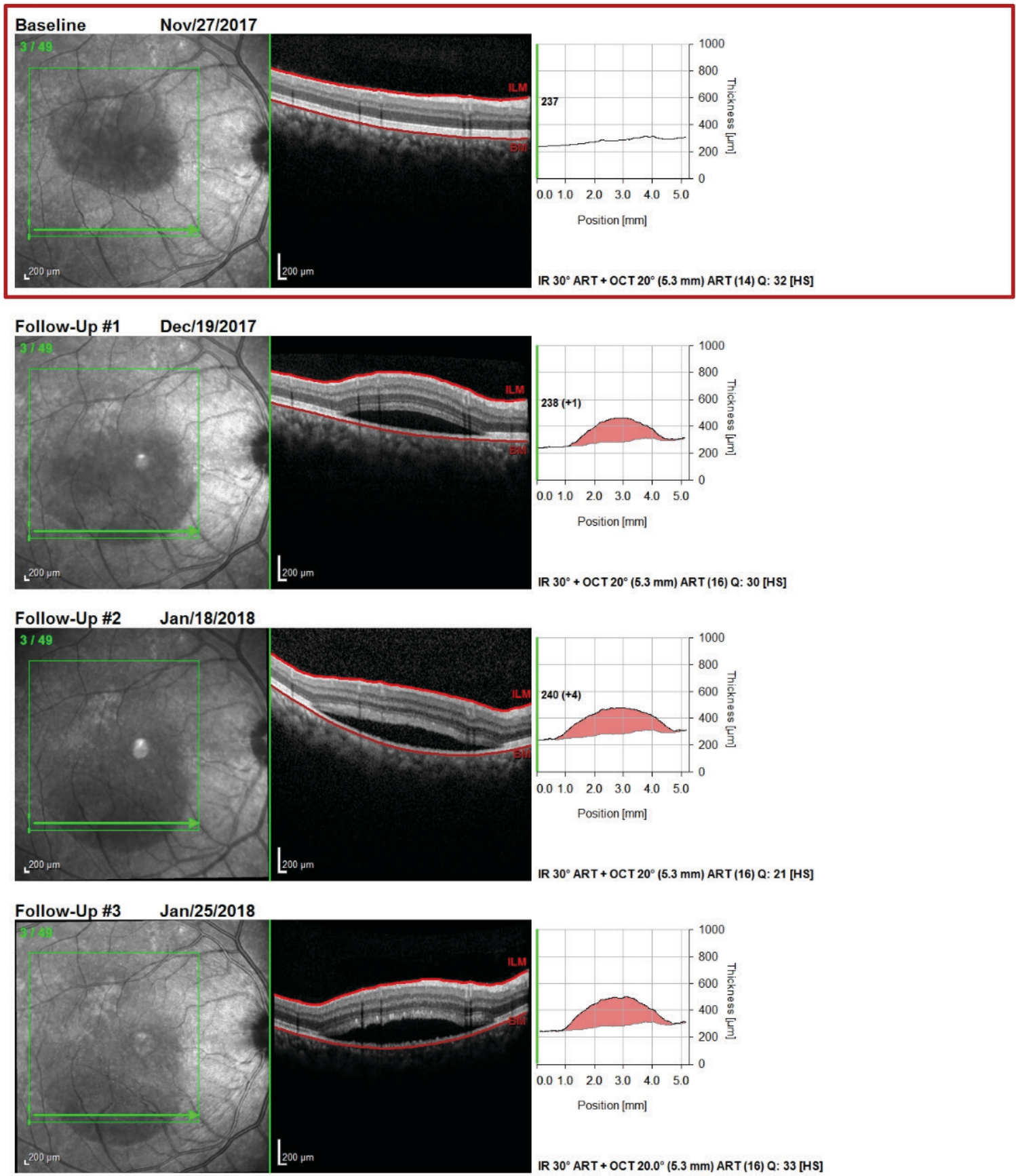
Figure 12 : B. Section horizontale traversant la fovéa montrant un liquide subrétinien persistant causant un décollement séreux progressif et une expansion de la rétine atteinte coïncidant avec une diminution nette de l'épaisseur maculaire.
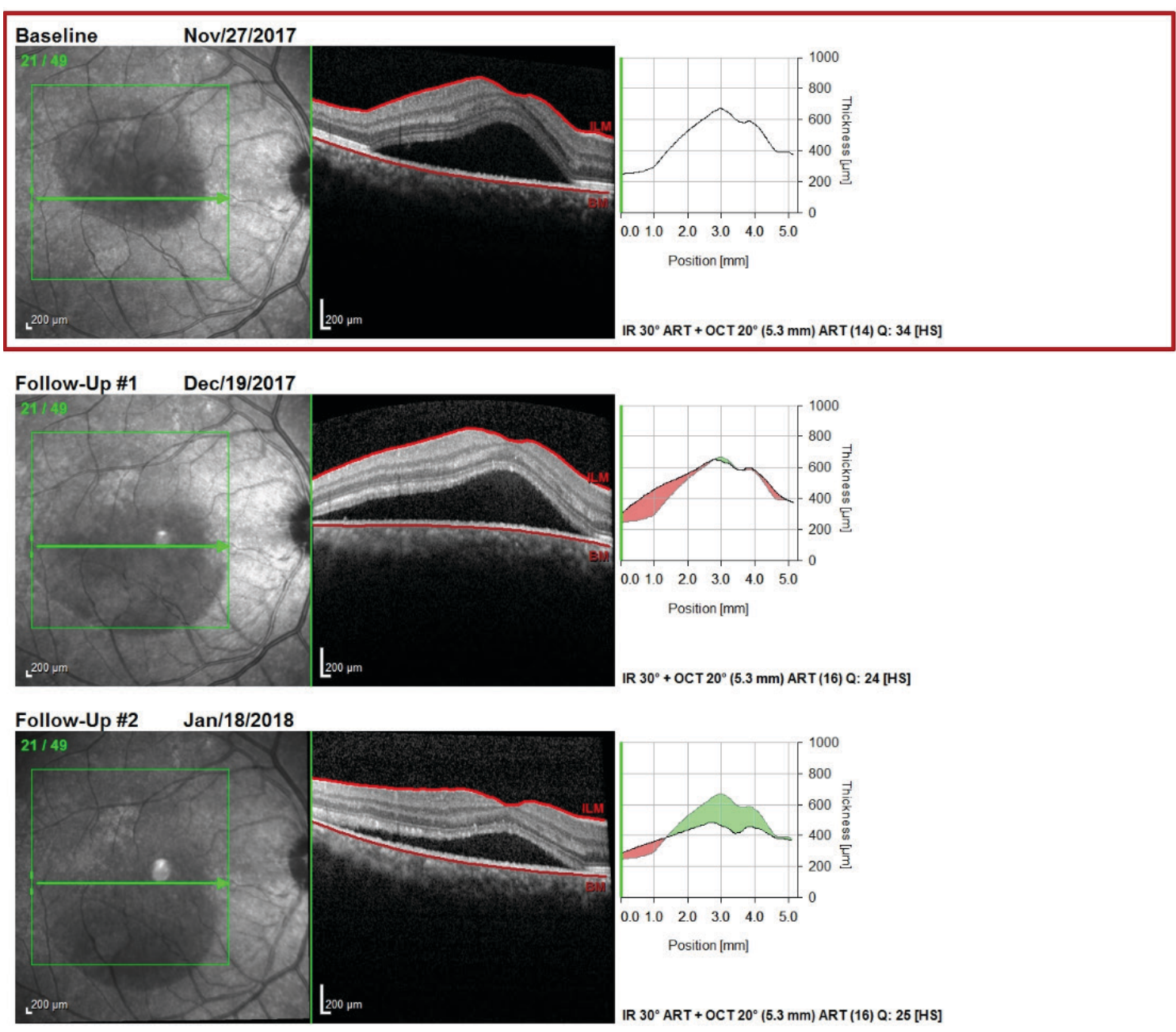

Follow-Up\#3 Jan/25/2018

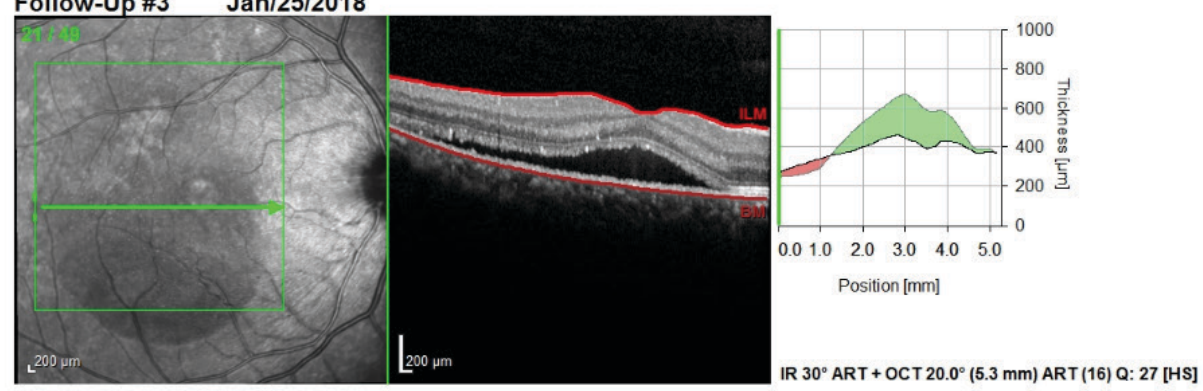


Le patient a reçu un diagnostic de choriorétinopathie séreuse centrale OD. Les antécédents du patient et les résultats de la TCO suggèrent un épisode aigu. Il a admis avoir récemment subi du stress à la maison et au travail, ce qui a peut-être précipité ses symptômes, et il a nié toute utilisation récente de stéroïdes. Il a été décidé d'observer le patient au moyen de l'imagerie par TCO en série.

Au cours des deux visites suivantes et sur une période de deux mois, les symptômes visuels du patient ont persisté sans amélioration. L'acuité visuelle est demeurée de 20/20 - OD, OS. La comparaison en série de l'imagerie TCO a démontré une migration infériorieuse du fluide, une constatation courante dans la CRSC, mais le liquide sous-rétinien central persistant est demeuré. En raison des symptômes du patient et de la persistance du liquide subrétinien central, le patient a commencé à prendre $50 \mathrm{mg}$ d'éplénone par voie orale pendant 4 semaines à sa deuxième visite après avoir vérifié qu'il ne prenait pas de médicament antihypertenseur à ce moment-là. Le traitement n'a pas encouragé la résorption des fluides à partir de l'imagerie par TCO en série et n’a pas non plus réduit les symptômes du patient.

Le patient devait se présenter à notre clinique d'angiographie fluorescéinique optométrique. L'étude d'angiographie a démontré une zone de regroupement focal précoce le long de l'arc temporel supérieur OD qui augmentait en fluorescence à travers les phases intermédiaires et tardives dans un modèle de tache d'encre (figure 13). La zone de regroupement correspondait au petit DEP de la TCO et a confirmé une rupture dans le complexe de l'EPR de la membrane de Bruch. En raison de la persistance de la maladie malgré l'observation et l'eplerénone, ainsi que de la frustration du patient et des troubles visuels subjectifs, le patient a été aiguillé vers l'ophtalmologie pour des options de traitement. Le patient ne s'est pas présenté pour un suivi.

Figure 13 : De gauche à droite, de haut en bas, on observe une augmentation de la fluorescence entre les premiers stades et les derniers stades de la formation de taches d'encre.
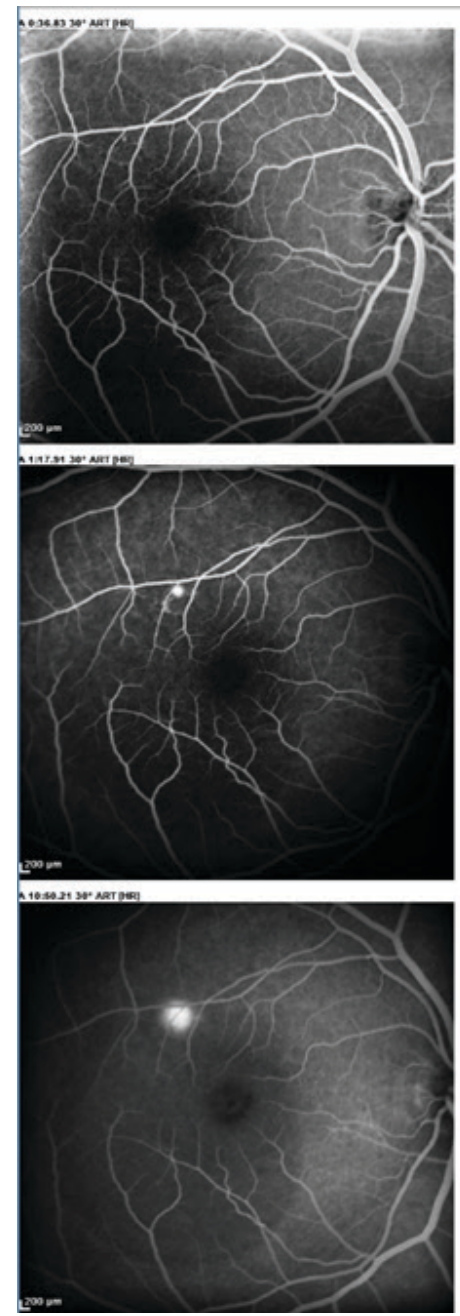
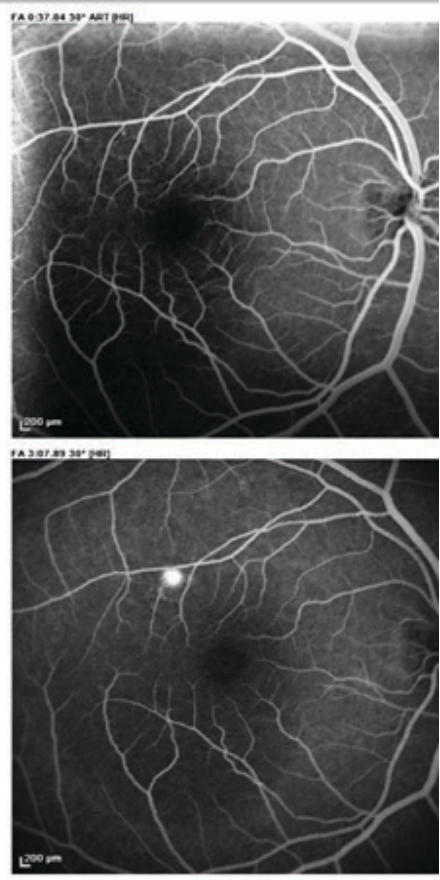
DISCUSSION

La pathophysiologie de la CRSC peut généralement être décrite comme une anomalie de la circulation choroïdienne entraînant une perturbation de l'EPR et de l'interaction neurosensorielle de la rétine. En cas de stase, de nonperfusion choriocapillaire ou d'inflammation, une altération de la pression hydrostatique peut perturber l'EPR, ce qui permet au liquide de pénétrer dans l'espace sous-rétinien et de causer un détachement neurosensoriel. Les détachements focaux de l'EPR (DEP), qui peuvent se présenter isolément ou éparpillés dans le pôle postérieur, sont souvent des cibles de traitement dans la coagulation laser focale pour réduire l'afflux de liquide subrétinien. ${ }^{21,22} \mathrm{Il}$ a été proposé que l'épaisseur choroïdienne accrue soit le résultat d'une augmentation de la perméabilité choroïdienne, ce qui entraîne un état hyperperméable qui est plus vulnérable aux fuites. ${ }^{5}$

Une hypothèse actuellement acceptée concernant le rôle des corticostéroïdes dans la CRSC indique que l'altération de l'activité des récepteurs glucocorticoïdes et minéralocorticoïdes dans le choroïde et l'EPR peut entraîner la rétention d'eau et de sodium, ce qui est responsable du détachement neurosensoriel séreux. ${ }^{23} \mathrm{Il}$ a été démontré que l'aldostérone, le principal minéralocorticoïde du corps, induit un stress oxydatif, une inflammation et un dysfonctionnement endothélial à de faibles doses et peut affecter l'expression dans les cellules gliales rétiniennes Muller dans l'échange d'ions et d'eau. ${ }^{23}$ L'injection intravitréenne de glucocorticoïdes n'a pas été associée à une incidence plus élevée de CRSC, ce qui suggère une étiologie métabolique d'ordre supérieur, bien qu'il y ait eu des cas signalés d'aggravation de CRSC préexistant avec triamcinolone intravitréen..$^{23,24}$

Dans le cas de CRSC aiguë, qui est communément défini comme étant la résolution dans les 3 à 4 mois suivants la présentation, les symptômes visuels soutenus sont souvent limités, puisque l'intégrité fovéale est préservée même en cas d'intervention fovéale. ${ }^{25}$ La prise en charge initiale des cas aigus, comme celui du patient $n^{\circ} 1$, est souvent observée. Dans une étude de 2017, Daruich et coll. ont évalué 31 patients n'ayant jamais reçu de traitement au cours de leur premier épisode, dont $84 \%$ avaient une résolution sans intervention de 6 mois. ${ }^{26}$ Après la résolution, toutefois, jusqu'à $50 \%$ des patients développent une récidive dans le même œil dans l'année suivant la présentation initiale et sont plus susceptibles de récidiver s'ils ont des antécédents de dépression ou de maladie psychiatrique. ${ }^{4}$ Les patients qui ont des antécédents de CRSC aiguë résolus peuvent encore présenter des séquelles visuelles comme la métamorphopsie et une vision de contraste et de couleur réduite en raison de la modification structurelle de la couche de photorécepteurs et de la réduction de la densité du cône. ${ }^{5}$ Ces dommages s'aggravent dans les cas chroniques et récurrents.

Si le liquide sous-rétinien persistant dure plus de 3 à 4 mois, le problème est considéré comme chronique et nécessite souvent une intervention. La séparation persistante de la rétine neurosensorielle entraîne l'atrophie des segments extérieurs de la couche photorécepteur, ce qui cause des dommages rétiniens externes permanents.? Les précipitations hyperréfléchissantes, comme celles observées à la figure 9 , résultent de cette atrophie du segment extérieur et sont caractéristiques d'une maladie chronique. On a théorisé qu'ils résultent de l'accumulation de protéines et de macrophages à partir des segments externes des phagocytos qui migrent de façon inférieure sous l'influence de la gravité. ${ }^{14}$ L'œdème maculaire bulleux cystö̈de, comme on l'a vu chez le patient $\mathrm{n}^{\circ} 1$, a été associé à une maladie de plus de cinq ans et est un indicateur pronostique négatif. ${ }^{27}$ La perte de couche rétinienne externe et les dépôts granulaires antérieurs à l'EPR sont également associés à un œè̀me chronique. La maladie chronique peut entraîner l'atrophie de l'EPR, se présentant comme des régions marquées d'hypofluorescence sur le FAF, également constatées chez le patient $\mathrm{n}^{\circ} 1$. La formation de membranes néovasculaires choroïdiennes peut également compliquer l'évolution de la maladie. Chez les patients qui ont une maladie rétinienne externe aussi étendue, le pronostic est médiocre malgré le traitement. Le traitement vise à accélérer la récupération visuelle en plus de prévenir les séquelles négatives à long terme.

Les médicaments administrés par voie orale ont été ciblés comme une méthode non invasive visant à raccourcir l'évolution de la maladie, avec des résultats variables. Parmi les inhibiteurs de l'aldostérone, on a tenu compte de la spironolactone et de l'eplerénone. Il s'agit d'antimineralocorticoïdes stéroïdes utilisés pour traiter l'insuffisance cardiaque chronique et l'hypertension. Ils agissent comme diurétiques en bloquant la liaison de l'aldostérone au récepteur minéralocorticoïde par inhibition compétitive et peuvent avoir une certaine application dans les cas aigus. ${ }^{21}$ Zucchiatti et coll., en 2018, ont affirmé que la résolution de l'éphérone était plus élevée et plus rapide que celle observée dans les cas de SCC aigu, bien qu'il s'agisse d'un petit échantillon $\left(n=27\right.$ yeux). ${ }^{28}$ Bien que ces résultats n'aient pas encore été corroborés dans d'autres publications, il peut s'agir d'une thérapie de première intention bénéfique chez les patients qui n'a pas été résolue en temps opportun. D’autres essais prospectifs randomisés et multicentriques seraient utiles pour guider ce traitement. 
D’autres médicaments administrés par voie orale ont été envisagés, notamment l'aspirine, le kétoconazole, le mifépristone, le finastéride, la rifampine et l'acétazolamide. ${ }^{29,30}$ Bien qu'il n'existe aucune preuve pour appuyer ces thérapies, une étude non aléatoire en mode libre réalisée par Caccavale et coll. en Italie a démontré qu'une dose quotidienne de $100 \mathrm{mg}$ d'aspirine pendant un mois, puis de $100 \mathrm{mg}$ un jour sur deux pendant 5 mois, a donné lieu à une récupération visuelle plus rapide et moins de récurrences comparées au groupe de contrôle. ${ }^{30}$ Étant donné que l'hypercoagulabilité accrue et l'agrégation plaquettaire ont été associées à un risque accru de CRSC, les auteurs ont suggéré un traitement de six mois à l'aspirine et à faible dose pour réduire le temps de résolution en raison de ses propriétés anti-agrégées.

L'utilisation d'agents intravitréens anti-VEGF a été envisagée pour le traitement de la CRSC. Bien qu'il y ait des avantages évidents à utiliser l'anti-VEGF chez les patients qui développent une MNV secondaire à la CRSC, l'utilisation de l'anti-VEGF pour le traitement de la maladie elle-même demeure controversée. Le fait qu'il n'y ait pas d'augmentation du niveau de VEGF dans le vitré des patients qui ont la CRSC demeure un point de litige pour ceux qui s'opposent à l'utilisation de l'anti-VEGF pour la CRSC. ${ }^{31}$ Une méta-analyse de Chung et coll., qui comprenait quatre études comparant le bevacizumab intravitréen à la surveillance, n'a pas permis de déterminer de façon concluante un avantage de l'anti-VEGF dans la CRSC aiguë ou chronique. ${ }^{31}$

L'utilisation de la TPD a été largement reconnue comme une méthode efficace de promotion de l'absorption du liquide subrétinien dans la CRSC en ciblant les anomalies vasculaires choroïdiennes par injection intraveineuse de la vertéphrine photosensible et l'utilisation d'un laser qui cible de façon sélective la verteporfin. Cela réduit au minimum les dommages collatéraux et peut être appliqué à des zones plus vastes et plus centrales que le laser focal. Dans une étude réalisée en 2018, une réponse anatomique était évidente dans 96 \% (n=39) des cas de CRSC chronique avec préservation de la couche nucléaire extérieure et de la zone ellipsoïde. ${ }^{22}$ L'application de la TPD est guidée par une angiographie fluorescéinique ou une angiographie verte par indocyanine pour cibler les zones de fuite.

Il a été démontré que le laser thermique focal ciblant les zones locales de fuites et de DEP était bénéfique pour la CRSC. Le traitement au laser est souvent guidé par l'angiographie au vert d'indocyanine ou de la fluorescéine. Bien qu'il y ait des preuves d'une diminution de la durée de réabsorption du liquide sous-rétinien, on ne sait pas s'il y a une diminution du taux de récidive. Un laser focal ne peut pas être appliqué à de grandes régions ou à des régions trop proches de la fovéa. ${ }^{22,29}$

\section{CONCLUSION}

Bien que la plupart des cas de CRSC aiguë soient autolimitatifs et ne nécessitent aucune intervention, les cas chroniques et récurrents peuvent entraîner une perte de vision permanente. Lorsqu'elle est appliquée de façon appropriée, l'intervention médicale peut aider à réduire la durée de la maladie et le taux de récidive, ce qui, en fin de compte, améliore les résultats visuels. L'utilisation de l'imagerie multimodale permet un diagnostic plus précis de la maladie en écartant d'autres causes de détachement rétinien séreux. De plus, il permet de déterminer la chronicité de la maladie et améliore la capacité d'adapter le traitement à chaque cas. La FAF, la TCO, l'angiographie fluorescéinique et l'angiographie VIC révèlent chacune un élément unique du tableau clinique et offrent une perspective physiologique inestimable pour déterminer à la fois le pronostic du patient en CRSC et la meilleure thérapie ciblée. Le praticien doit être conscient des diverses options de traitement disponibles et aiguiller de façon appropriée les patients, surtout dans les cas chroniques, afin d'éviter les complications menaçant la vue.•

REMERCIEMENTS

Les auteurs tiennent à remercier Andrew Rixon, $\mathrm{OD}, \mathrm{FAAO}$, pour son soutien et ses conseils pendant la préparation de ce manuscrit.

DIVULGATIONS FINANCIÈRES

Les auteurs n'ont aucun intérêt financier et ne déclarent aucun conflit d'intérêts dans ce travail. 


\section{RÉFÉRENCES}

1. Liegl R, Ulbig MW. Central serous chorioretinopathy. Ophthalmologica 2014;232(2):65-76.

2. Schachat AP, ed. Ryan's Retina. Sixth edition. Edinburgh ; New York: Elsevier; 2018.

3. Wang M, Munch IC, Hasler PW, Prünte C, Larsen M. Central serous chorioretinopathy. Acta Ophthalmol 2008;86(2):126-145.

4. Liew G, Quin G, Gillies M, Fraser-Bell S. Central serous chorioretinopathy: a review of epidemiology and pathophysiology: Central serous chorioretinopathy. Clin Exp Ophthalmol 2013;41(2):201-214

5. Iacono P, Battaglia Parodi M, Falcomatà B, Bandello F. Central serous chorioretinopathy treatments: a mini review. Ophthalmic Res 2015;55(2):76-83.

6. Rasheed M, Goud A, Mohamed A, Vupparaboina K, Chhablani J. Change in choroidal vascularity in acute central serous chorioretinopathy. Indian J Ophthalmol 2018;66(4):530.

7. Ambiya V, Goud A, Rasheed MA, Gangakhedkar S, Vupparaboina KK, Chhablani J. Retinal and choroidal changes in steroid-associated central serous chorioretinopathy. Int J Retina Vitreous 2018;4(1).

8. Ersoz MG, Karacorlu M, Arf S, Hocaoglu M, Sayman Muslubas I. Pachychoroid pigment epitheliopathy in fellow eyes of patients with unilateral central serous chorioretinopathy. Br J Ophthalmol 2018 Apr;102(4):473-478.

9. Seo EJ, Um T, Yoon YH. Abnormal choroidal flow on optical coherence tomography angiography in central serous chorioretinopathy. Clin Exp Ophthalmol 2018 Dec 5. doi: 10.1111/ceo.13454. [Epub ahead of print].

10. Entezari M, Karimi S, Ramezani A, Nikkhah H, Fekri Y, Kheiri B. Choroidal thickness in healthy subjects. J Ophthalmic Vis Res 2018;13(1):39.

11. Margolis R, Spaide RF. A pilot study of enhanced depth imaging optical coherence tomography of the choroid in normal eyes. Am J Ophthalmol 2009;147(5):811-815

12. Chung Y-R, Kim JW, Choi S-Y, Park SW, Kim JH, Lee K. Subfoveal choroidal thickness and vascular diameter in active and resolved central serous chorioretinopathy. Retina 2018;38(1):102-107.

13. Matsumoto H, Kishi S, Otani T, Sato T. Elongation of photoreceptor outer segment in central serous chorioretinopathy. Am J Ophthalmol 2008;145(1):162-168.el.

14. Matsumoto H, Kishi S, Sato T, Mukai R. Fundus autofluorescence of elongated photoreceptor outer segments in central serous chorioretinopathy. Am J Ophthalmol 2011;151(4):617-623.el.

15. Bonini Filho MA, de Carlo TE, Ferrara D, et al. Association of choroidal neovascularization and central serous chorioretinopathy with optical coherence tomography angiography. JAMA Ophthalmol 2015;133(8):899.

16. Spaide R, Klancnikjr J. Fundus autofluorescence and central serous chorioretinopathy. Ophthalmology 2005;112(5):825-833.

17. Yung M, Klufas MA, Sarraf D. Clinical applications of fundus autofluorescence in retinal disease. Int J Retina Vitreous. 2016;2(1).
18. Ficker L, Vafidis G, While A, Leaver P. Long-term follow-up of a prospective trial of argon laser photocoagulation in the treatment of central serous retinopathy. Br J Ophthalmol 1988;72(11):829-834.

19. Chhablani J, Rani PK, Mathai A, Jalali S, Kozak I. Navigated foca laser photocoagulation for central serous chorioretinopathy. Clin Ophthalmol 2014 Aug 18;8:1543-1547.

20. Ambiya V, Khodani M, Goud A, et al. Early focal laser photocoagulation in acute central serous chorioretinopathy: a prospective, randomized study. Ophthalmic Surg Lasers Imaging Retina 2017 Jul 1;48(7):564-571.

21. Abouammoh MA. Advances in the treatment of central serous chorioretinopathy. Saudi J Ophthalmol 2015;29(4):278-286.

22. Ruiz-del-Tiempo MP, Calvo P, Ferreras A, Leciñena J, Pablo L, Ruiz-Moreno O. Anatomical retinal changes after photodynamic therapy in chronic central serous chorioretinopathy. J Ophthalmology 2018;2018:1-4.

23. Daruich A, Matet A, Dirani A, et al. Central serous chorioretinopathy: Recent findings and new physiopathology hypothesis. Prog Retin Eye Res 2015 Sep;48:82-118.

24. Zhao M, Bousquet E, Valamanesh F, et al. Differential regulation of AQP4 and Kir4.1 by triamcinolone acetonide and dexamethasone in the healthy and inflamed retina. Invest Opthalmol Vis Sci 2011;52(9):6340.

25. Wong KH, Lau KP, Chhablani J, Tao Y, Li Q, Wong IY. Central serous chorioretinopathy: what we have learnt so far. Acta Ophthalmol 2016;94(4):321-325.

26. Daruich A, Matet A, Marchionno L, et al. Acute central serous chorioretinopathy: factors influencing episode duration. Retina 2017;37(10):1905-1915.

27. Do JL, Olmos de Koo LC, Ameri H. Atypical chronic central serous chorioretinopathy with cystoid macular edema: Therapeutic response to medical and laser therapy. J Curr Ophthalmol 2017;29(2):133-135

28. Zucchiatti I, Sacconi R, Parravano MC, et al. Eplerenone versus observation in the treatment of acute central serous chorioretinopathy: a retrospective controlled study. Ophthalmol Ther 2018 Jun;7(1):109-118.

29. Nicholson B, Noble J, Forooghian F, Meyerle C. Central serous chorioretinopathy: update on pathophysiology and treatment. Surv Ophthalmol 2013;58(2):103-126.

30. Caccavale A, Romanazzi F, Imparato M, Negri A, Morano A, Ferentini F. Low-dose aspirin as treatment for central serous chorioretinopathy. Clin Ophthalmol 2010;4:899-903.

31. Chung Y-R, Seo EJ, Lew HM, Lee KH. Lack of positive effect of intravitreal bevacizumab in central serous chorioretinopathy: metaanalysis and review. Eye 2013;27(12):1339-1346. 